\title{
A novel device for the study of somatosensory information processing
}

\author{
Jameson K. Holden, Richard H. Nguyen, Eric M. Francisco, Zheng Zhang, Robert G. \\ Dennis, and Mark Tommerdahl \\ Department of Biomedical Engineering, University of North Carolina at Chapel Hill, Chapel Hill, \\ NC 27599, United States
}

\begin{abstract}
Current methods for applying multi-site vibratory stimuli to the skin typically involve the use of multiple, individual vibrotactile stimulators. Limitations of such an arrangement include difficulty with both positioning the stimuli as well as ensuring that stimuli are delivered in a synchronized and deliberate manner. Previously, we reported a two-site tactile stimulator that was developed in order to solve these problems (Tannan et al., 2007a). Due to both the success of that novel stimulator and the limitations that were inherent in that device, we designed and fabricated a foursite stimulator that provides a number of advantages over the previous version. First, the device can stimulate four independent skin sites and is primarily designed for stimulating the digit tips. Second, the positioning of the probe tips has been re-designed to provide better ergonomic hand placement. Third, the device is much more portable than the previously-reported stimulator. Fourth, the stimulator head has a much smaller footprint on the table or surface where it resides. To demonstrate the capacity of the device for delivering tactile stimulation at four independent sites, a finger agnosia protocol, in the presence and absence of conditioning stimuli, was conducted on seventeen healthy control subjects. The study demonstrated that with increasing amplitudes of vibrotactile conditioning stimuli concurrent with the agnosia test, inaccuracies of digit identification increased, particularly at digits D3 and D4. The results are consistent with prior studies that implicated synchronization of adjacent and near-adjacent cortical ensembles with conditioning stimuli in impacting TOJ performance (Tommerdahl et al., 2007).
\end{abstract}

\section{Keywords}

Somatosensory; Vibrotactile; Tactile stimulator; Agnosia; Synchronization

\section{INTRODUCTION}

For the past several years, our research group has been working towards the development of a portable tactile stimulator that could effectively be used to study changes in sensory information processing in clinical and clinical research venues across a diverse spectrum of neurological disorders. Thus far, we have gone through several iterations in the development of this stimulator. The first prototype of the device (Tannan et al., 2005a) was used to demonstrate changes in spatial acuity with repetitive stimulation. A subsequent report described that this change did not occur with individuals with autism, strongly suggesting a

\footnotetext{
(C) 2011 Elsevier B.V. All rights reserved.

Publisher's Disclaimer: This is a PDF file of an unedited manuscript that has been accepted for publication. As a service to our customers we are providing this early version of the manuscript. The manuscript will undergo copyediting, typesetting, and review of the resulting proof before it is published in its final citable form. Please note that during the production process errors may be discovered which could affect the content, and all legal disclaimers that apply to the journal pertain.
} 
lower-than-normal inhibitory response (Tommerdahl et al., 2007a). A second iteration of the device (Tannan et al., 2007a) was much more portable as well as more robust and reliable in its ability to deliver well-controlled vibrotactile stimuli to the skin. The device proved extremely useful, and a number of studies were conducted with it that demonstrated the ability to reliably and reproducibly obtain metrics of neuro-adaptation (Tannan et al., 2007b), temporal order judgment (TOJ) and the impact of synchronized conditioning stimuli on TOJ (Tommerdahl et al., 2007b), the absence of the impact of those same conditioning stimuli on TOJ in individuals with autism (Tommerdahl et al., 2008), the relationship between spatial acuity and amplitude discrimination (Zhang et al., 2008), a method for the study of tactile-thermal interactions (Zhang, 2009), a reliable means for measuring amplitude discriminative capacity and a robust near-linear relationship between duration of repetitive conditioning stimuli and the impact of that conditioning on amplitude discriminative capacity (Tannan et al., 2007b), the below-normal adaptation metrics in autism (Tommerdahl et al., 2007), the impact of NMDA receptor block on adaption metrics (Folger et al., 2009), a demonstration of Weber's law (Francisco et al., 2008; Holden et al., 2011) and a robust relationship with neurophysiological data (Francisco et al., 2008), and differences in timing perception in Parkinson's Disease (Nelson et al., 2011). More recently, we have developed a newer, more portable and ergonomic model of the device, which is much more suited for a clinical or clinical research environment, and is capable of delivering vibrotactile stimuli to four fingers: the index (D2), middle (D3), ring (D4), and little (D5) fingers. The utility of this device has been recently reported in a paper that reported phenotypic differences within a spectrum of patients with vulvodynia (Zhang et al., 2011), and in a paper that describes its utility for describing phenotypic differences within the autism spectrum via modulating vibrotactile stimuli (i.e., sinusoidal stimuli that dynamically change in amplitude), but the device itself, as well as a demonstration of its capability to deliver four-digit protocols, has not been fully described, which is the purpose of this report. In a subsequent paper, a magnet-compatible version of this device will be reported.

\section{METHODS}

\subsection{Hardware}

The Cortical Metrics (CM-4; see Figure 1) stimulator was developed in our laboratories for use in series of experiments such as those described in this report. The system was designed using state-of-the-art rapid manufacturing technology to allow multiple identical systems to be built and used in different locations. Also, the use of rapid manufacturing permitted very rapid design evolution, thereby potentiating the production of special fixtures and changes to geometry as needed for special applications. The device consists of two separate parts: the main body and a detachable head unit. The flat plates of all exterior housing and other components of approximately planar geometry are direct manufactured using lasermachined $6 \mathrm{~mm}$ acrylic sheet, cut on a 120 Watt $\mathrm{CO}_{2}$ laser engraving system, model number X660 (Universal Laser Systems, Scottsdale, AZ). The more complex housing and internal mechanism components are direct manufactured from ABS plus, by fusion deposition modeling (FDM) on a StrataSys Dimension bst 1200es (StrataSys, Inc., Eden Prairie, MN). The cylindrical trays forming the disks of the head unit are CNC machined from 1" thick Acetal (Delrin) plate. All housing and mechanism components and assemblies were solid modeled prior to fabrication using SolidWorks solid modeling software (SolidWorks Corporation, Concord, MA).

The internal mechanism of the head unit is comprised of identical cylindrical disks placed sideways and four abreast (130 $\mathrm{mm}$ in diameter, $11 \mathrm{~mm}$ in depth) between two plastic supports. Each disk can be independently rotated to adjust for differing finger lengths for each test subject. A voice coil actuator (VCA) and an optical position sensor are mounted in each disk. Each VCA is attached to a plastic probe $(5 \mathrm{~mm}$ diameter) which slightly protrudes 
through a hole ( $7 \mathrm{~mm}$ diameter) in the side of the cylinder. The amount of protrusion for each probe is independently adjustable as are the positions of the holes to accommodate the length of the subject's fingers. The VCAs drive the plastic stimulator probe tips according to prescribed sinusoidal waveforms. The moving components of the stimulator tips are directly manufactured from Polycarbonate (PC) by 3-D FDM as a single compliant mechanism component integrating a mounting flange, a thin-beam four-bar linkage, a magnet coil bobbin, an optical displacement sensor vane, and the extension to the mechanical stimulator tip. The compliant four-bar linkage mechanism allows the coil, optical position sensor vane, and tip to be displaced vertically along a straight line for a distance of $\pm 1 \mathrm{~mm}$. The 4-bar compliant mechanism also provides a very low hysteresis linear restoring force to center each tip vertically when no current is applied to the VCA coil. The VCA coil is 400 turns of 34 AWG magnet wire (approximately $30 \mathrm{Ohms}$ total resistance), wrapped in a rectangular bobbin permanently solvent bonded into the four-bar mechanism. The entire four-bar mechanism is $5.3 \mathrm{~mm}$ in thickness, and is positioned such that the VCA coils sit directly between two opposed rectangular N42 rare-earth-element magnets (catalog number BCC2, $\mathrm{K} \& \mathrm{~J}$ Magnetics, Jamison, PA) similar to those found in computer hard drives. The resulting VCA motors generate extremely linear force outputs as a function of drive current with very low hysteresis due to the "frictionless" nature of the single-piece bearing-less four-bar compliant mechanism. The position of the vibrating tips is detected by noncontacting optical displacement sensors, one for each tip, similar in configuration to ones we have previously employed in precision optical force transducers (Dennis and Kosnik, 2002). When the tips are not being driven, the optical position sensors can act as a highly-sensitive contact or force sensor. By employing the optical position sensor, the tips can be driven to contact the skin, and the contact force of each tip can be adjusted independently due to the fact that the spring constant of each VCA four-bar linkage mechanism is identical.

The custom electronics were designed using free CAD software from ExpressPCB (www.expresspcb.com). The printed circuit boards were manufactured using the resulting CAD files, also by ExpressPCB. The hybrid circuit includes signal amplifiers for the position sensors, an analog controller to allow either "force" or "position" control of each VCA motor and tip, a tunable analog PID controller for position control of each tip, and a bipolar push-pull high-current op-amp output stage to drive each VCA motor. This hybrid circuit is interfaced via four parallel pin connectors ( 2 banks of 50 pins for digital signals and 2 banks of 34 pins for analog signals) to an internal NI-USB-6259 data acquisition (DAQ) board. The DAQ board then interfaces via a USB connection to any standard PC running Microsoft Windows XP or later.

\subsection{Software}

A custom line-of-business application was developed for the Microsoft. Net platform using the C\# programming language and Windows Presentation Foundation (WPF) framework to control the stimulator and administer the data collection protocols. The interface was designed to be intuitive, extensible, and aesthetically pleasing. The software needed to be extensible to facilitate the development of future protocols for a device as flexible as the CM-4. The core extensibility was achieved by using a "plugin" architecture with a shell application whose function is to discover, load and execute small plugins. The shell exposes a software contract (an inheritable C\# class) that is consumed and extended by each plugin. Each task described in this paper represents one such plugin. Most traditional neuropsychological protocols using the standard X-alternative forced-choice (X-AFC) tracking method (Cornsweet, 1962) can be created with only a couple dozen lines of C\# code. While most plugins interact directly with the CM-4 stimulator, this is not a requirement of the plugin contract. Plugins can, for example, be designed to collect arbitrary subject information pertinent to the given study (e.g. participant demographics, relevant 
medical history, various surveys, etc.). The net effect is not only a significant reduction in the amount of clinical paperwork that needs to be completed by each participant, but also a marked reduction in data-entry time for clinicians. All data collected by the application are stored in an encrypted (128-bit RC4) SQLite database in a user-specified location. Each database can be shared with multiple instances of the shell application, providing a mechanism for seamless networking of CM-4 stations (Holden, et al, 2011). The software is also capable of storing, as well as creating and customizing, all relevant initialization information for each plugin, such that a given battery of protocols can be administered repeatedly and in a consistent manner, while maintaining flexibility for future projects. The batteries allow for greater reuse of each plugin, resulting in shorter development times a more efficient workflow throughout an experiment.

\subsection{Protocols}

In order to demonstrate exemplary use of the CM-4, a finger agnosia test, in the presence and absence of conditioning stimuli, was performed. The finger agnosia test was designed to assess the capacity of subjects to recognize and identify stimulated digits, an assessment similar to tactile finger recognition or localization tests (Boll, 1974; Reitan and Wolfson, 1993) utilized in current neuropsychological diagnostics.

\subsection{Subjects}

Seventeen healthy subjects (8 males and 9 females), ranging from 22 to 57 (39.1 \pm 2.9 ) years of age, were recruited for the study. None of the subjects reported any neuropsychological impairment and all were naïve to both the study design and issue under investigation. The study was performed in accordance with the Declaration of Helsinki, all subjects gave their informed consent, and the experimental procedures were reviewed and approved in advance by an institutional review board.

\subsection{Experimental Procedure}

During an experimental session, the subjects were seated comfortably in a chair with the right arm resting on the device. Because the lengths of fingers typically vary among subjects, the positions of the probe tips were individually adjusted to ensure that they contacted the glabrous, padded tips of the fingers of each subject. These loci were chosen in order to allow the convenience of access and comfort of participants as well as for the wealth of neurophysiologic information that exists for the corresponding somatotopic regions of cortex in primates (Chen et al., 2003, 2007, 2009; Francisco et al., 2008; Friedman et al., 2008; LaMotte and Mountcastle, 1975; Mountcastle, 1969; Tommerdahl et al., 1993, 1998, 2002, 2005, 2006, 2010). As depicted in Figure 1, probe tip positioning was accomplished by loosening a set screw and rotating each of the drums independently to conform to the natural hand shape of each subject. After proper positioning, if the probe tips still failed to make proper contact with the digits, the tips themselves were either raised or lowered. Once adjusted, the probe tips were locked in place prior to initiation of the battery so that they would remain immobile during testing. At the start of each run, the four tips were driven towards the tips of the fingers in order to ensure good contact with the skin.

During the assessment, the device delivered constant-amplitude sinusoidal skin displacements (vibrations) via flat Delrin probes $(5-10 \mathrm{~mm}$ in diameter) positioned to make contact with the tips of the index (D2), middle (D3), ring (D4), and little (D5) fingers of the right hand. The independent probe tips were computer-controlled and capable of delivering a wide range of vibrotactile stimulation of varying frequencies $(\mathrm{Hz})$ and amplitudes $(\mu \mathrm{m})$. Stimulus parameters were specified by test algorithms that were based on specific protocols as well as subject responses during those protocols. 
Subjects viewed a computer monitor that provided continuous visual cueing during the experimental session. Specifically, an onscreen light panel indicated to the participant when stimuli were being delivered and when subjects were to respond. Training trials were not included prior to testing, and the subjects were not given performance feedback or knowledge of the results during data acquisition. The sensory testing session was conducted by application of low frequency $(25 \mathrm{~Hz})$ vibration to selected fingers. Each battery of testing lasted between 15 and 20 minutes depending on the protocols being run and on subject performance. Each individual protocol typically lasted 2 to 3 minutes.

\subsection{Finger Agnosia Protocol}

Finger agnosia tests are typically utilized to diagnose the ability of subjects to recognize and identify stimulated digits (Boll, 1974; Reitan and Wolfson, 1993). In order to assess the ability of the subject to discriminate one digit from another, a four-alternative forced-choice (4-AFC) protocol was implemented. Figure 2 represents a timeline for the finger agnosia protocols evaluated. The device delivered a short pulse or tap ( $300 \mu \mathrm{m}, 25 \mathrm{~Hz}, 40 \mathrm{~ms})$ to one of the four digits in a pseudo-random order on a trial-by-trial basis, and subjects were queried as to which digit was stimulated (Figure 2). The simple test was used in order to determine baseline values for each subject. A more complex agnosia test was subsequently administered in which test stimuli were delivered to the skin as a tap as in the previous test ( $300 \mu \mathrm{m}, 25 \mathrm{~Hz}, 40 \mathrm{~ms}$ ), but in the presence of conditioning stimuli at variable amplitudes. In each case, a $25 \mathrm{~Hz}, 500 \mathrm{~ms}$ conditioning stimulus was delivered to all four digits at one of four amplitudes: 30, 40, 50, and $100 \mu \mathrm{m}$. The conditioning stimulus was delivered $500 \mathrm{~ms}$ prior to, and $500 \mathrm{~ms}$ following, the tap of the test digit (Figure 2). For all finger agnosia tasks, subjects indicated which finger was perceived to have received the large amplitude tap by choosing the respective digit on an image of the dorsal side of a hand presented on a computer monitor. Test stimuli sites were pseudo-randomized on a trial-by-trial basis. The subjects were assessed on their accuracy over a total of 16 trials (4 trials for each digit as the test stimulus).

\subsection{Analysis}

For the finger agnosia protocols, accuracy percentages were calculated by analyzing the ratio of correct to total responses of the subjects. Percent accuracies were trial-independent and reflected accuracies across all 16 trials. The $100 \mu \mathrm{m}$ conditioning condition was chosen for further analysis because of the significantly lower percent accuracy compared to the simple agnosia task. Percent inaccuracies were quantified for the $100 \mu \mathrm{m}$ conditioning stimulus by calculating the frequency at which digits were incorrectly chosen. Results were calculated in this manner in order to compare percent inaccuracies with difference limens (DLs), where lower value might suggest higher accuracies and increased discriminative capabilities. The data were analyzed for significance by calculating $\mathrm{p}$-values across mean inaccuracy metrics for each digit. Histograms were plotted in order to visualize the differences among each of the digits with respect to standard error of the means. Statistical ttests were used to evaluate the difference of the performance of each subject under different conditions. A probability value of less than 0.05 was considered statistically significant.

\subsection{Auditory Cue Analysis}

To ensure that the stimulator did not produce any audible cues during the agnosia task, an auditory output analysis was conducted using a standard USB microphone and the open source software suite Audacity. The microphone was placed on a table $31 \mathrm{~cm}$ from the stimulator head unit. Four one-second recordings were created with each condition consisting of an initial $250 \mathrm{~ms}$ period of silence followed by a single-channel $300 \mu \mathrm{m} 25 \mathrm{hz}$ sinusoidal vibration lasting $500 \mathrm{~ms}$ and ending with another $250 \mathrm{~ms}$ period of silence. Audacity provides a contrast analysis tool in compliance with the Web Content Accessibility 
Guidelines (WCAG 2.0), Success Criteria 1.4.7. This tool was used to calculate the RMS amplitude in decibels $(\mathrm{dB})$ during each vibration and period of silence.

\section{RESULTS}

This study employed a finger agnosia protocol, in the presence and absence of conditioning stimulation, on healthy subjects in order to demonstrate the capacity of the device for delivering well-controlled vibrotactile stimuli at four independent sites. Additionally, an auditory cue analysis was performed in order to ensure that there were no auditory cues available for the subjects during the finger agnosia protocol. The auditory cue analysis found no indication of any auditory cues being produced by the stimulator during a vibration. The peak amplitude for any channel during a vibration was $-42.64 \mathrm{~dB}$ (silence is considered to be in the range of $-30 \mathrm{~dB}$ ). The average RMS amplitude (during all vibrations) was $-58.90 \pm 0.11$ $\mathrm{dB}$. The average RMS amplitude during the periods of silence was $-58.83 \pm 0.10 \mathrm{~dB}$. Comparing each condition's vibration to the immediately preceding silence yielded an average difference in RMS amplitude of $0.05 \pm 0.13 \mathrm{~dB}$.

The finger agnosia task was evaluated in order to quantify the ability of subjects to recognize and identify stimulated digits in the absence and in the presence of conditioning stimuli at different amplitudes. This task included seventeen healthy subjects ( 8 males and 9 females) ranging from 22 to $57(39.1 \pm 2.9)$ years of age. As shown in Figure 3, the average percent accuracy in the absence of conditioning stimuli was $98.2 \pm 0.9 \%(\mathrm{n}=17)$, and accuracy across subjects decreased with increasing amplitude of conditioning stimuli. Conditioning amplitudes of 30 and $40 \mu \mathrm{m}$ resulted in percent accuracies of $93.7 \pm 3.0 \%$ and $94.9 \pm 4.2 \%$, respectively, and were not statistically significant compared to subject performance in the absence of conditioning stimuli. The effect of conditioning on the finger agnosia task became statistically significant at conditioning amplitudes greater than $50 \mu \mathrm{m}$ : $89.0 \pm 4.2 \%$ at $50 \mu \mathrm{m}(\mathrm{p}<0.06)$ and $60.0 \pm 7.6 \%$ at $100 \mu \mathrm{m}(\mathrm{p}<0.01)$. Because the conditioning stimuli at $100 \mu \mathrm{m}$ resulted in the most significant percentage of incorrect responses compared to the simple finger agnosia protocol, the frequency of inaccurate responses for each digit was quantified (Figure 4). The results suggested that subjects, on average, made the largest number of inaccurate responses when the correct answer should have been D3 and D4 (percent inaccuracies of $60.0 \pm 10.0 \%$ and $55.0 \pm 14.6 \%$, respectively). Subjects were relatively better at identifying stimulation of D2 (inaccuracy of $15.0 \pm 10.0 \%$ significantly better than that for $\mathrm{D} 3, \mathrm{p}<0.01$ ) and better at identifying $\mathrm{D} 5$, though not statistically significantly more.

\section{DISCUSSION}

The delivery of sinusoidal displacements to a single skin site via mechanical transducer has been used extensively for the study of flutter vibration in both psychophysical and neurophysiological settings for a number of decades. Exemplary uses of such a device are described in Goble and Hollins, 1993; Juliano et al., 1989; LaMotte and Mountcastle, 1975; Mountcastle et a., 1969; Tannan et al., 2006; Tommerdahl et al., 1993, 1998, 2002; and Vierck and Jones, 1970. Typically, stimuli that can be delivered through mechanical transducers - vertical displacement stimulators such as the one originally described by Chubbuck (1966) - were used for studies of somatosensation and are very well equipped to deliver sinusoidal stimuli at a frequency range (1 to $250 \mathrm{~Hz}$ ) with amplitudes of sufficient size (between 0 and $1000 \mu \mathrm{m}$ ) to activate a broad range of mechanoreceptors. However, in order to stimulate more than one skin site - either during the course of human psychophysical testing or animal experimentation - it is necessary to position a second vertical displacement stimulator over the second skin site. Our previous device (described in Tannan et al. 2007a) was designed to address this issue by allowing dual site stimulation 
with automated two-dimensional probe positioning. Although the device reported by Tannan and colleagues was successfully utilized in a number of studies (Tannan et al., 2005b, 2006, 2007b, 2008; Tommerdahl et al., 2007a, 2007b, 2008), it was cumbersome and not ideal for clinical and clinical research venues. The CM-4, described in this report, has the capacity to quickly and easily adjust to fit to most adult, and many juvenile, hand sizes and can deliver vibrotactile stimuli to the tips of four digits. The ability to simultaneously deliver vibrotactile stimuli to a number of digits allows for a great deal of protocol diversity.

In this report, we described a relatively simple four-site finger agnosia protocol to demonstrate the potential utility of the device. The principle finding in the results of this study is that there is an increase in inaccuracies with increases in the amplitude of concurrent conditioning stimulation delivered during the agnosia task, and the ability to perform the task accurately in the presence of that conditioning stimulation is diminished more in digits D3 and D4 than in digits D2 and D5. The decrease in accuracy with increasing amplitudes of synchronized sinusoidal stimulation is consistent with prior reports of increasing inaccuracies in temporal order judgment (TOJ) in the presence of synchronized and periodic conditioning stimuli. In a study by Tommerdahl and colleagues (Tommerdahl et al., 2007), it was demonstrated that TOJ results obtained from a number of pairs of stimulus sites - unilateral as well as bilateral - were comparable. However, in the presence of a $25 \mathrm{~Hz}$ conditioning sinusoidal stimulus which was delivered both before, concurrently and after the TOJ task, there was a significant increase in the TOJ measured when the two stimuli were located unilaterally on digits D2 and D3. In the presence of the same $25 \mathrm{~Hz}$ conditioning stimulus, the TOJ obtained when the two stimuli were delivered bilaterally was not impacted. This led to the speculation that the impact that the conditioning stimuli which only had an impact if they were sinusoidal, periodic and synchronous - had on TOJ measures was due to the synchronization of adjacent cortical ensembles in somatosensory cortex, and that the synchronization of these cortical ensembles could have been responsible for the degradation in temporal order judgment. The conditioning stimuli in this study were also synchronized, periodic and simultaneous, and if the degradation in test performance was due to synchronization of adjacent cortical ensembles similar to what was speculated in the TOJ report, then inaccuracies due to this synchronization would be lower on the digits on the perimeter of the cortical ensemble (i.e., D2 and D5), and the results reflect this prediction. Future studies will consider whether or not subjects with neurological disorders are not impacted by conditioning stimuli, as was found to be the case in subsequent TOJ studies (e.g., TOJ metrics of subjects with autism were not impacted significantly by conditioning stimuli; Tommerdahl et al., 2008).

The degree of inaccuracies in the different digits with increasing conditioning stimulation is also consistent with motor studies of digit interdependencies. In studying the autonomy of finger movements, intended motion in one finger often results in simultaneous movement, or enslavement, of other digits. More specifically, D3 and D4 show the most enslavement, or interdependency, of adjacent digits while D2 is characterized by the greatest independence (Häger-Ross and Schieber, 2000). In observing motor-related cortical potentials (MRCPs), the autonomous nature of D2 was shown to be significantly high while D4 showed the most dependency on other digits (Slobounov et al., 2002). In Figure 4, D2 demonstrates the lowest inaccuracies in the presence of conditioning stimulation while D3 and D4 exhibit the most; thus, in both the motor and sensory based studies, D2 demonstrates the most independence.

The role of neural communication between adjacent and non-adjacent cortical regions plays an important role in understanding the relationship between neurophysiological mechanisms and sensory percept. The development of new, more versatile devices and methodologies, such as presented in this report, could contribute to bridging decades of neuroscientific 
research with human perceptual clinical and clinical research studies. One long term goal of our research is to develop sensory based instrumentation and methodologies for the diagnosis and assessment of treatment efficacies for a broad range of neurological disorders, and building this aforementioned bridge could provide new insights into fundamental information processing mechanisms as well as generating perceptual metrics that are more sensitive to alterations in central information processing capacity.

\section{Acknowledgments}

This work was supported, in part, by the Department of Defense, Congressionally Directed Medical Research Program (CDMRP) W81XWH-07-1-0287 and NIH 1-R21-NS072811-01A1.

\section{References}

Boll TJ. Right and left cerebral hemisphere damage and tactile perception: Performance of the ipsilateral and contralateral sides of the body. Neuropsychologia. 1974; 12:235-238. [PubMed: 4842916]

Chen LM, Friedman RM, Row AW. Optical Imaging of a Tactile Illusion in Area $3 b$ of the Primary Somatosensory Cortex. Science. 2003; 302:881-885. [PubMed: 14500850]

Chen LM, Friedman RM, Row AW. Optical Imaging of Digit Topography in Individual Awake and Anesthetized Squirrel Monkeys. Exp Brain Res. 2009; 196:393-401. [PubMed: 19484466]

Chen LM, Turner GH, Friedman RM, Zhang N, Gore JC, Roe AW, Avison MJ. High-Resolution Maps of Real and Illusory Tactile Activation in Primary Somatosensory Cortex in Individual Monkeys with Functional Magnetic Resonance Imaging and Optical Imaging. J Neurosci. 2007; 27(34):91819191. [PubMed: 17715354]

Chubbuck JG. Small-motion biological stimulator. APL Tech Digest. 1966:18-23.

Cornsweet TN. The staircase-method in psychophysics. Am J Psychol. 1962; 75:485-491. [PubMed: 13881416]

Dennis, RG.; Kosnik, PE. Mesenchymal cell culture: instrumentation and methods for evaluating engineered muscle. In: Atala, A.; Lanza, R., editors. Methods in Tissue Engineering. 2002. p. 307-316.

Folger SE, Tannan V, Zhang Z, Holden JK, Tommerdahl M. Effects of N-methyl-D-aspartate receptor antagonist dextromethorphan on vibrotactile adaptation. BMC Neurosci. 2008; 16(9):87. [PubMed: 18796147]

Francisco E, Tannan V, Zhang Z, Holden J, Tommerdahl M. Vibrotactile amplitude discrimination capacity parallels magnitude changes in somatosensory cortex and follows Weber's Law. Exp Brain Res. 2008; 191(1):49-56. [PubMed: 18651137]

Friedman RM, Chen LM, Row AW. Responses of Areas 3b and 1 in Anesthetized Squirrel Monkeys to Single- and Dual-Site Stimulation of the Digits. J Neurophysiol. 2008; 100:3185-3196. [PubMed: 18922955]

Goble AK, Hollins M. Vibrotactile adaptation enhances amplitude discrimination. J Acoust Soc Am. 1993; 93(1):418-424. [PubMed: 8423258]

Häger-Ross C, Schieber MH. Quantifying the Independence of Human Finger Movements: Comparisons of Digits, Hands, and Movement Frequencies. J Neurosci. 2000; 20(22):8542-8550. [PubMed: 11069962]

Holden JK, Francisco EM, Zhang Z, Baric C, Tommerdahl M. An undergraduate laboratory exercise to study Weber's Law. J Undergrad Neurosci Edu. 9(2):A71-A74.

Juliano SL, Whitsel BL, Tommerdahl M, Cheema SS. Determinants of patchy metabolic labeling in the somatosensory cortex of cats: a possible role for intrinsic inhibitory circuitry. J Neurosci. 1989; 9(1):1-12. [PubMed: 2913199]

LaMotte RH, Mountcastle VB. Capacities of humans and monkeys to discriminate vibratory stimuli of different frequency and amplitude: a correlation between neural events and psychological measurements. J Neurophysiol. 1975; 38(3):539-559. [PubMed: 1127456] 
Mountcastle VB, Talbot WH, Sakata H, Hyvärinen J. Cortical neuronal mechanisms in fluttervibration studied in unanesthetized monkeys. Neuronal periodicity and frequency discrimination. J Neurophysiol. 1969; 32(3):452-484. [PubMed: 4977839]

Nelson TS, Suhr CL, Lai A, Halliday AJ, Freestone DR, McLean KJ, Burkitt AN, Cook MJ. Exploring the tolerability of spatiotemporally complex electrical stimulation paradigms. Epilepsy Res. 2011

Reitan, RM.; Wolfson, D. The Halstead-Reitan neuropsychological test battery: Theory and clinical interpretation. 2. Tuscon AZ: Neuropsychology Press;

Slobounov S, Johnston J, Chiang H, Ray WJ. Motor-related Cortical Potentials Accompanying Enslaving Effect In Single Versos Combination of Fingers Force Production Tasks. Clinical Neurophysiology. 2002; 113:1444-1452. [PubMed: 12169327]

Tannan V, Dennis R, Tommerdahl M. A novel device for delivering two-site vibrotactile stimuli to the skin. J Neurosci Methods. 2005a; 147(2):75-81. [PubMed: 15970331]

Tannan V, Dennis RG, Tommerdahl M. Stimulus-dependent effects on tactile spatial acuity. Behav Brain Funct. 2005b; 1:18. [PubMed: 16216121]

Tannan V, Dennis RG, Zhang Z, Tommerdahl M. A portable tactile sensory diagnostic device. J Neurosci Methods. 2007a; 164(1):131-138. [PubMed: 17537516]

Tannan V, Holden JK, Zhang Z, Baranek GT, Tommerdahl MA. Perceptual metrics of individuals with autism provide evidence for disinhibition. Autism Res. 2008; 1(4):223-230. [PubMed: 19360672]

Tannan V, Simons S, Dennis RG, Tommerdahl M. Effects of adaptation on the capacity to differentiate simultaneously delivered dual-site vibrotactile stimuli. Brain Res. 2007b; 1186:164170. [PubMed: 18005946]

Tannan V, Whitsel BL, Tommerdahl MA. Vibrotactile adaptation enhances spatial localization. Brain Res. 2006; 1102(1):109-116. [PubMed: 16806126]

Tommerdahl M, Delemos KA, Favorov OV, Metz CB, Vierck CJ Jr, Whitsel BL. Response of anterior parietal cortex to different modes of same-site skin stimulation. J Neurophysiol. 1998; 80(6):32723283. [PubMed: 9862921]

Tommerdahl M, Favorov OV, Whitsel BL. Dynamic Representations of the Somatosensory Cortex. Neuroscience and Biobehavioral Reviews. 2010; 34:160-170. [PubMed: 19732790]

Tommerdahl M, Favorov OV, Whitsel BL. Effects of High-Frequency Skin Stimulation on SI Cortex: Mechanisms and Functional Implication. Somatosensory and Motor Research. 2005; 22(3):151160. [PubMed: 16338824]

Tommerdahl M, Favorov O, Whitsel BL. Optical imaging of intrinsic signals in somatosensory cortex. Behav Brain Res. 2002; 135(1-2):83-91. [PubMed: 12356438]

Tommerdahl M, Favorov O, Whitsel BL, Nakhle B, Gonchar YA. Minicolumnar activation patterns in cat and monkey SI cortex. Cereb Cortex. 1993; 3(5):399-411. [PubMed: 8260808]

Tommerdahl M, Simons SB, Chiu JS, Favorov O, Whitsel BL. Ipsilateral input modifies the primary somatosensory cortex response to contralateral skin flutter. J Neurosci. 2006; 26(22):5970-5977. [PubMed: 16738239]

Tommerdahl M, Tannan V, Cascio CJ, Baranek GT, Whitsel BL. Vibrotactile adaptation fails to enhance spatial localization in adults with autism. Brain Res. 2007a; 1154:116-123. [PubMed: 17498672]

Tommerdahl M, Tannan V, Holden JK, Baranek GT. Absence of stimulus-driven synchronization effects on sensory perception in autism: Evidence for local underconnectivity? Behav Brain Funct. 2008; 24(4):19. [PubMed: 18435849]

Tommerdahl M, Tannan V, Zachek M, Holden JK, Favorov OV. Effects of stimulus-driven synchronization on sensory perception. Behav Brain Funct. 2007b; 4(3):61.

Vierck CJ Jr, Jones MB. Influences of low and high frequency oscillation upon spatio-tactile resolution. Physiol Behav. 1970; 5(12):1431-1435. [PubMed: 5524531]

Zhang Z, Tannan V, Holden JK, Dennis RG, Tommerdahl M. A quantitative method for determining spatial discriminative capacity. Biomed Eng Online. 2008; 10(7):12. [PubMed: 18331644]

Zhang Z, Francisco EM, Holden JK, Dennis RG, Tommerdahl M. The impact of non-noxious heat on tactile information processing. Brain Res. 2009; 1302:97-105. [PubMed: 19765551] 
Zhang Z, Zolnoun DA, Francisco EM, Holden JK, Dennis RG, Tommerdahl M. Altered central sensitization in subgroups of women with vulvodynia. Clin J Pain. 2011; 27(9):755-63. [PubMed: 21593667]

\section{Abbreviations}

TOJ Temporal Order Judgment

NMDA N-methyl-D-aspartate

FDM Fusion Deposition Modeling

VCA Voice Coil Actuator

CAD Computer-Aided Design

AFC Alternative-Forced Choice

DL Difference Limen 


\section{Highlights}

- A new portable sensory based tactile device is described.

- The portable device is capable of stimulating 4 finger tips independently which allows for the study of cortical-cortical interactions and their direct impact on sensory percepts.

- Conditioning stimuli have a profound, yet predictable, impact on simple tactile tests in healthy individuals. 


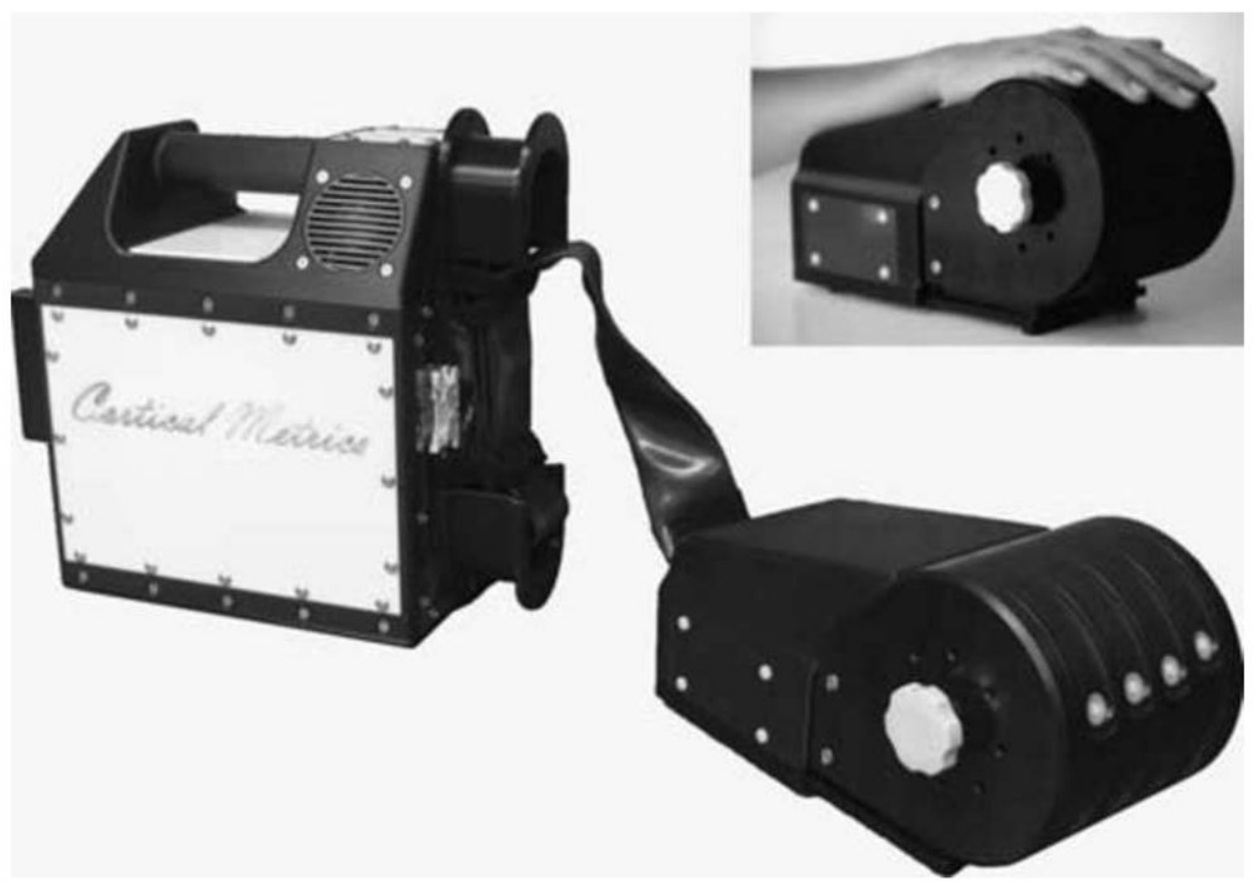

Figure 1.

Four Site Vibrotactile Stimulator. Each of the four probe tips is positioned by rotating the four independently-positioned drums to maximize contact between finger pads and the stimulator tips. During an experimental session, subjects were seated comfortably in a chair with their arm resting on the arm rest attached to the head unit of the device. Digits D2 through D5 were then positioned for vibrotactile stimulation. 


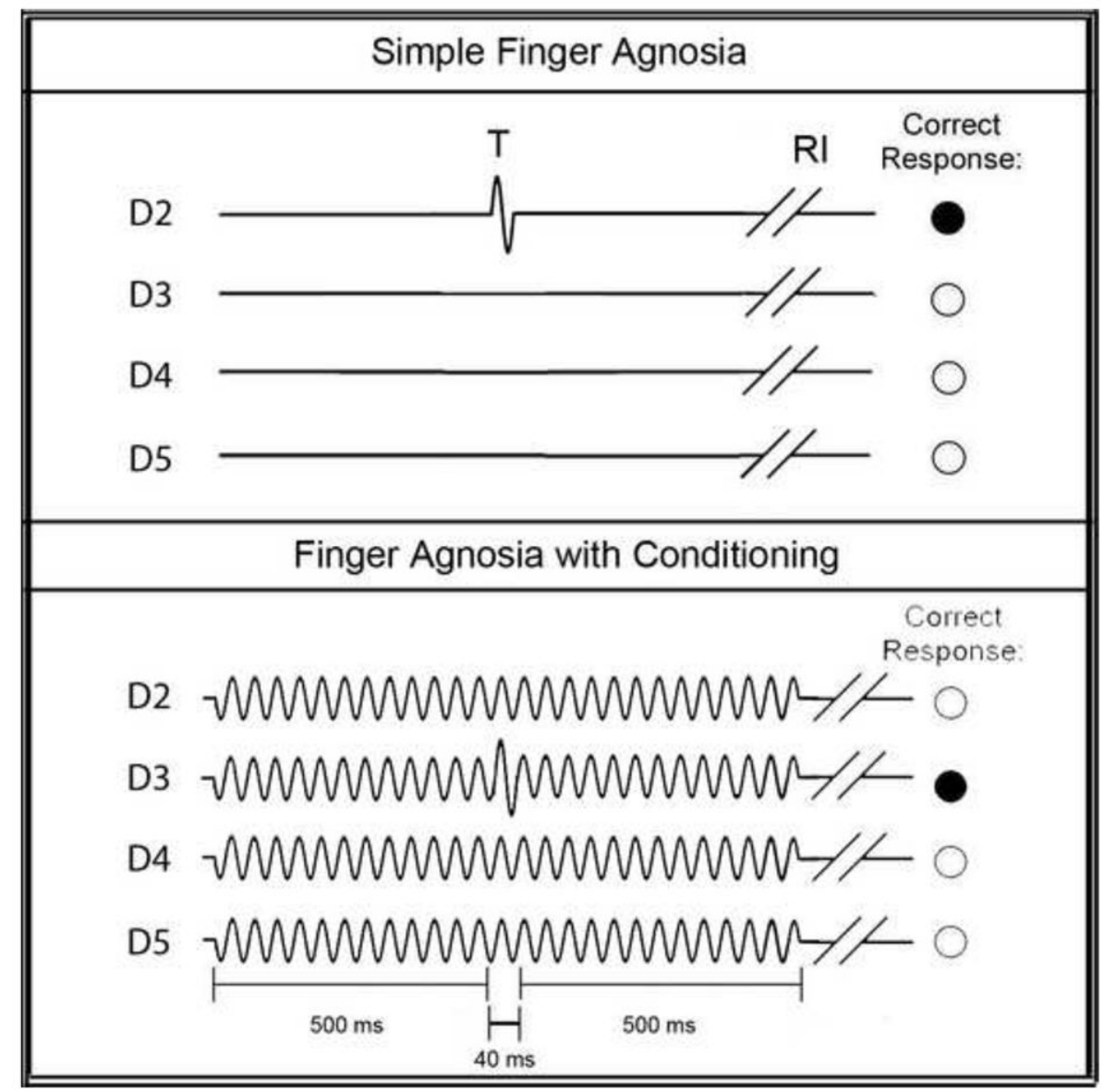

Figure 2.

Schematics of Finger Agnosia Protocols. The simple finger agnosia assessment (top panel) consisted of a 4AFC protocol where a short test (T) pulse $(300 \mu \mathrm{m}, 25 \mathrm{~Hz}, 40 \mathrm{~ms})$ was delivered to one of the four digits followed by a subject response interval (RI). The finger agnosia test was also conducted in the presence of conditioning stimuli of amplitudes 30, 40, 50 , or $100 \mu \mathrm{m}$ (bottom panel). The conditioning stimulus was delivered $500 \mathrm{~ms}$ prior to, and $500 \mathrm{~ms}$ following, the tap of the test digit. For all finger agnosia tasks, subjects indicated which finger was perceived to have received the large amplitude tap by choosing the respective digit on an image of the dorsal side of a hand presented on a computer monitor. Test stimuli sites were pseudo-randomized on a trial-by-trial basis. 


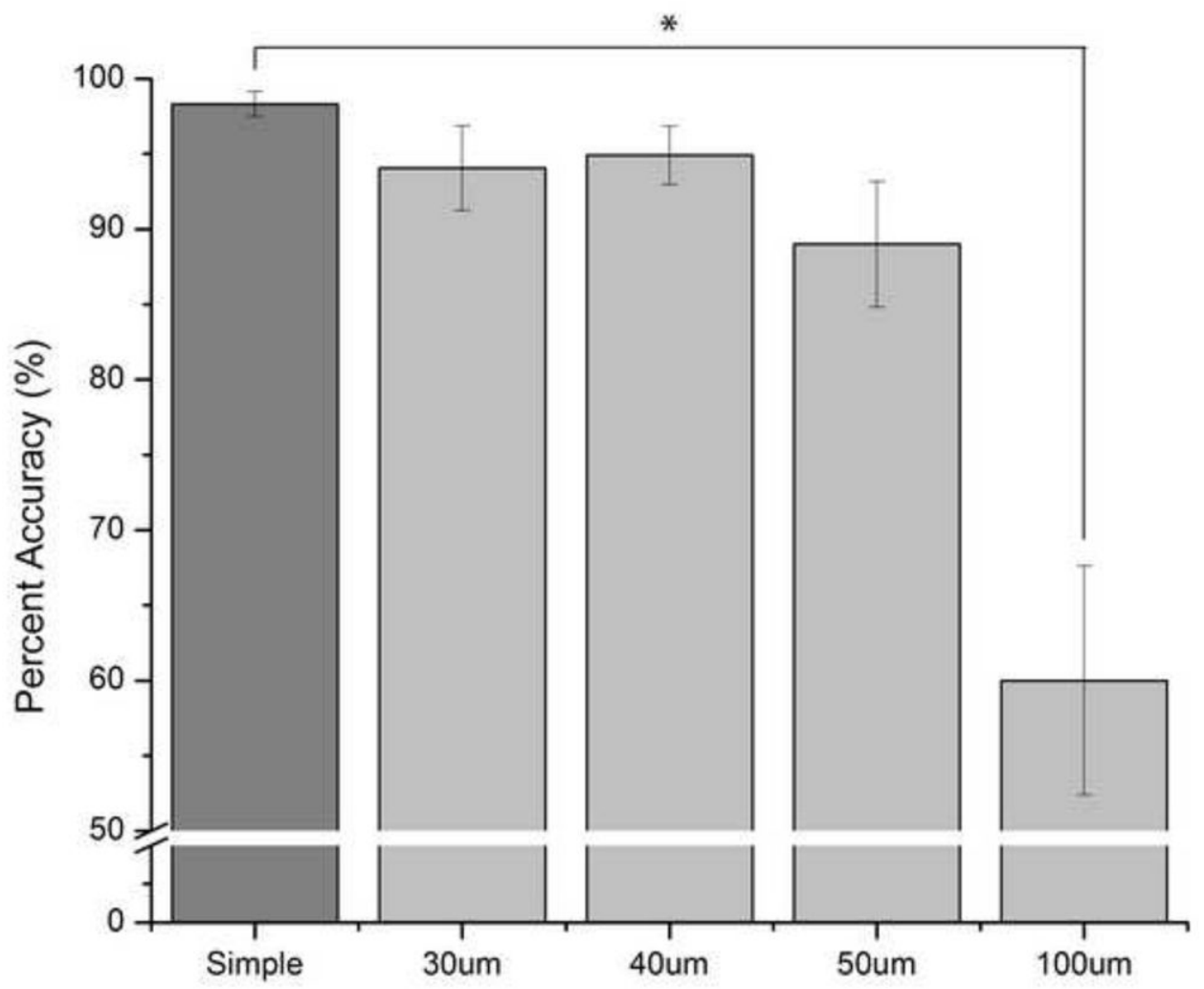

Figure 3.

Average Percent Accuracy the Effect of Conditioning Stimuli on the Finger Agnosia Task. The average percent accuracy in absence of conditioning stimuli was $98.2 \pm 0.9 \%(n=17)$. In the presence of $30,40,50$, and $100 \mu \mathrm{m}$ conditioning stimuli, the percent accuracies gradually decreased with increased amplitude of conditioning stimuli: $93.7 \pm 3.0 \%$ at $30 \mu \mathrm{m}$ $(\mathrm{n}=17), 94.9 \pm 4.2 \%$ at $40 \mu \mathrm{m}(\mathrm{n}=16), 89.0 \pm 4.2 \%$ at $50 \mu \mathrm{m}(\mathrm{n}=17, \mathrm{p}<0.06)$, and $60.0 \pm 7.6 \%$ at $100 \mu \mathrm{m}(\mathrm{n}=5, \mathrm{p}<0.01)$. 


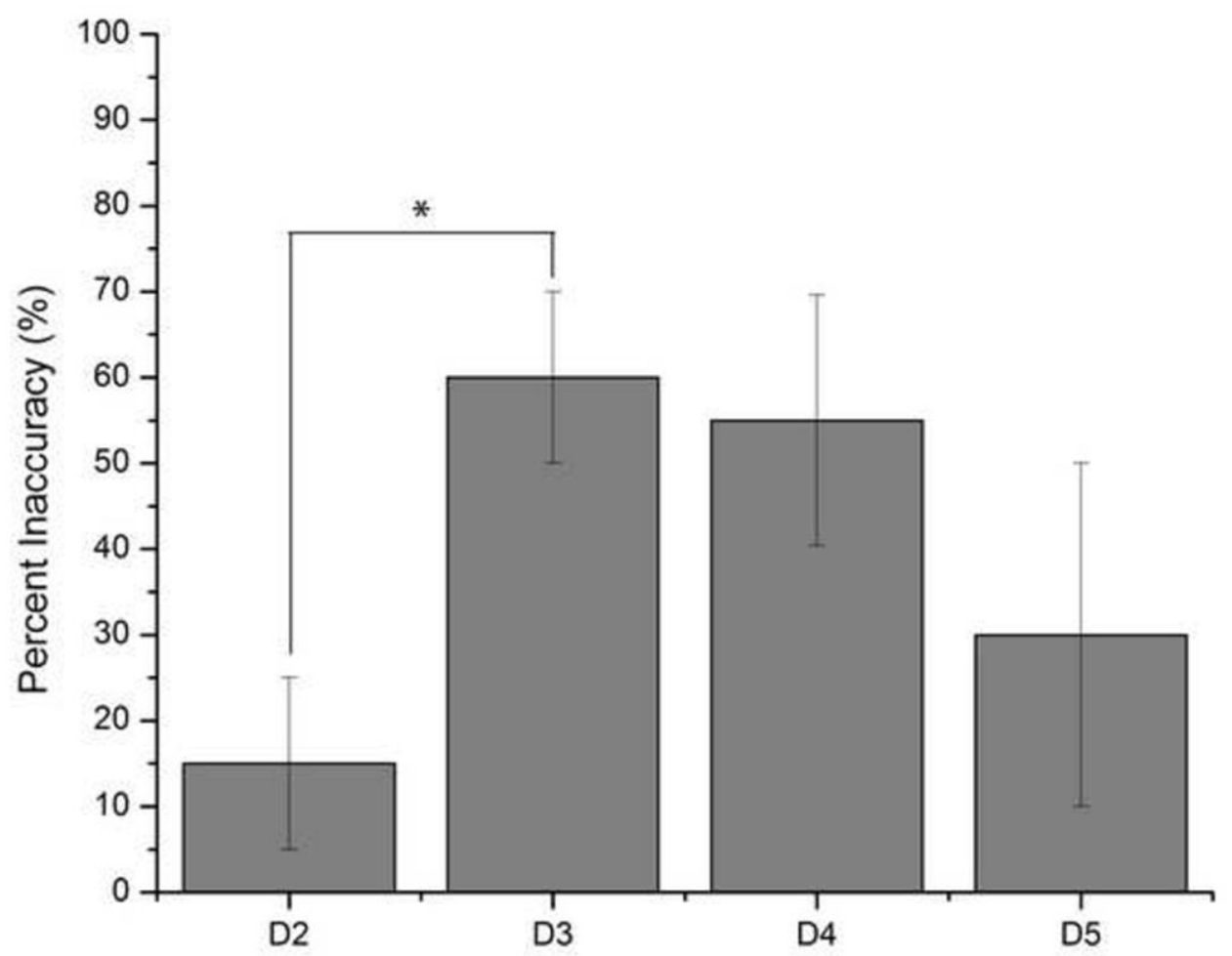

Figure 4.

Average Percent Inaccuracy on Finger Agnosia with $100 \mu \mathrm{m}$ Conditioning Stimuli. Digits D3 and D4 showed the highest percent inaccuracies of $60.0 \pm 10.0 \%$ and $55.0 \pm 14.6 \%$, respectively. There was a statistically significant observation in accurately recognizing and identifying stimulation of D2 at $15.0 \pm 10.0 \%$ versus D3 at $30.0 \pm 20.0 \%(\mathrm{p}<0.01)$ and slight discrimination difference between D2 and D4 ( $<<0.08)$ in the presence of the $100 \mu \mathrm{m}$ conditioning stimuli. The other the digit combinations showed no statistical significance in discrimination capability. 